\title{
Reconfigurable Surface Micropatterns Based on the Magnetic Field-Induced Shape Memory Effect in Magnetoactive Elastomers
}

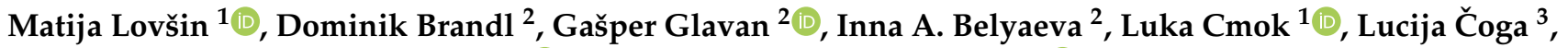 \\ Mitjan Kalin ${ }^{3}$, Mikhail Shamonin ${ }^{2}(\mathbb{D})$ and Irena Drevenšek-Olenik ${ }^{1,4, *(\mathbb{D}}$ \\ 1 Department of Complex Matter, J. Stefan Institute, Jamova 39, 1000 Ljubljana, Slovenia; \\ matija.lovsin@ijs.si (M.L.); luka.cmok@ijs.si (L.C.) \\ 2 East Bavarian Centre for Intelligent Materials (EBACIM), Ostbayerische Technische Hochschule (OTH) \\ Regensburg, Seybothstr. 2, 93053 Regensburg, Germany; dominik1.brandl@st.oth-regensburg.de (D.B.); \\ gasper.glavan@oth-regensburg.de (G.G.); inna.belyaeva@oth-regensburg.de (I.A.B.); \\ mikhail.chamonine@oth-regensburg.de (M.S.) \\ 3 Faculty of Mechanical Engineering, University of Ljubljana, Aškerčeva 6, 1000 Ljubljana, Slovenia; \\ Lucija.Coga@fs.uni-lj.si (L.Č.); mitjan.kalin@fs.uni-lj.si (M.K.) \\ 4 Faculty of Mathematics and Physics, University of Ljubljana, Jadranska 19, 1000 Ljubljana, Slovenia \\ * Correspondence: irena.drevensek@ijs.si
}

\section{check for}

updates

Citation: Lovšin, M.; Brandl, D.; Glavan, G.; Belyaeva, I.A.; Cmok, L.; Čoga, L.; Kalin, M.; Shamonin, M.; Drevenšek-Olenik, I. Reconfigurable Surface Micropatterns Based on the Magnetic Field-Induced Shape Memory Effect in Magnetoactive Elastomers. Polymers 2021, 13, 4422 https://doi.org/10.3390/ polym13244422

Academic Editor: Marcin Masłowski

Received: 3 December 2021

Accepted: 14 December 2021

Published: 16 December 2021

Publisher's Note: MDPI stays neutral with regard to jurisdictional claims in published maps and institutional affiliations.

Copyright: (c) 2021 by the authors. Licensee MDPI, Basel, Switzerland. This article is an open access article distributed under the terms and conditions of the Creative Commons Attribution (CC BY) license (https:// creativecommons.org/licenses/by/ $4.0 /)$.
Abstract: A surface relief grating with a period of $30 \mu \mathrm{m}$ is embossed onto the surface of magnetoactive elastomer (MAE) samples in the presence of a moderate magnetic field of about $180 \mathrm{mT}$. The grating, which is represented as a set of parallel stripes with two different amplitude reflectivity coefficients, is detected via diffraction of a laser beam in the reflection configuration. Due to the magnetic-field-induced plasticity effect, the grating persists on the MAE surface for at least $90 \mathrm{~h}$ if the magnetic field remains present. When the magnetic field is removed, the diffraction efficiency vanishes in a few minutes. The described effect is much more pronounced in MAE samples with larger content of iron filler (80 wt $\%$ ) than in the samples with lower content of iron filler $(70 \mathrm{wt} \%)$. A simple theoretical model is proposed to describe the observed dependence of the diffraction efficiency on the applied magnetic field. Possible applications of MAEs as magnetically reconfigurable diffractive optical elements are discussed. It is proposed that the described experimental method can be used as a convenient tool for investigations of the dynamics of magnetically induced plasticity of MAEs on the micrometer scale.

Keywords: magnetoactive elastomers; shape memory effect; surface microstructuring; optical diffraction

\section{Introduction}

Surface properties play a vital role in many physical processes. They govern interfacial phenomena, such as friction, adhesion and wettability. They also determine the interactive properties of the objects with optical radiation, like objects' color appearance and shine. One of the essential surface features is surface topography. Practically any mechanism that possesses a capacity to modify surface topography also affects the phenomena mentioned above.

Magnetoactive elastomers (MAEs) are rubbery substances composed of a compliant polymer matrix with embedded micrometer-sized ferromagnetic particles [1-5]. They are also known as magnetorheological elastomers (MREs) and are often perceived as solid analogs to magnetorheological fluids [6-8]. The exposure of MAEs to a magnetic field is an intriguing process that can induce profound changes in the surface topography of a compliant material [9,10]. In soft MAEs with Young's modulus below $100 \mathrm{kPa}$ (in the absence of a magnetic field) [11-13], a magnetic field applied in the direction perpendicular to the surface induces the formation of "mountain-like" structures [14], which resemble the 
Rosensweig peaks in ferrofluids and produce a strong increase in surface roughness [15-24]. In our recent characterization of this effect, we found that the root mean square (RMS) roughness was in the range of $1 \mu \mathrm{m} / \mathrm{T}$ [25]. Other phenomena on MAE surfaces that were also demonstrated to be regulable by a magnetic field are wettability $[14,15,26-28]$, adhesive properties [29-32], friction [33-38], drop splashing [39] and surface optical properties [21]. For the latter, it was demonstrated that the total optical reflectivity and the type of reflection (specular or diffuse) could be controlled by the magnetic field.

Exposure of MAEs to magnetic fields also provokes the appearance of another interesting phenomenon, namely the magnetically induced plasticity effect, also known as the magnetic shape memory effect $[5,40-46]$. This effect provides a possibility to temporary "freeze" the shape of a MAE object by exposing it to the magnetic field [47]. It also enables to temporary create different patterns in the MAE surface by the embossing process $[48,49]$. The embossed pattern persists while the magnetic field is present and fades away when the field is removed. The recovery happens due to the regained elasticity of the material that flattens the surface back to its initial shape. Afterward, another pattern can be imposed.

Up to now, investigations of the shape memory effect in MAEs have been limited to macroscopic forms and patterns (size of several $\mathrm{mm}$ ). In this work, we report on the magnetic regulation of a micropattern embossed in the MAE surface. The pattern had a periodic structure with the periodicity of $30 \mu \mathrm{m}$, due to which it could have been conveniently detected via diffraction of a laser beam. Its stability in a constant magnetic field, erasure with decreasing magnetic field and reconfigurability after the erasure process were analyzed. In addition, being a convenient tool for probing the micropatterning features, the explored optical diffraction is also interesting due to its possible applications in devices such as magnetically reconfigurable diffractive optical elements (DOEs), magnetically tunable optical sensors and magneto-optical feedback units.

\section{Materials and Methods}

The disk-shaped MAE samples with a diameter of $20 \mathrm{~mm}$ and a thickness of $2 \mathrm{~mm}$ were synthesized following the previously described synthesis routes [12,50]. The compositions containing 70 and $80 \mathrm{wt} \%$ of soft-magnetic carbonyl iron powder (CIP, type SQ, BASF SE Carbonyl Iron Powder \& Metal Systems, Ludwigshafen, Germany) and exhibiting different stiffness in zero magnetic field were investigated. The incorporated CIP particles had a mean diameter of $4.5 \mu \mathrm{m}$ and were uniformly distributed in the polymer matrix. Stamps for embossing were fabricated from the standard photoresist material (SU-8) that was spin-coated onto a glass plate and then structured into a pattern of periodic slabs by a laser direct imaging system (ProtoLaser LDI, LPFK Laser \& Electronics, Garbsen, Germany). The slabs had a quadratic cross-section of $15 \times 15 \mu \mathrm{m}^{2}$ and were separated laterally for $15 \mu \mathrm{m}$ from each other. Hence, they formed a one-dimensional periodic structure with the periodicity $\Lambda=30 \mu \mathrm{m}$. The patterned area had a size of $20 \times 4 \mathrm{~mm}^{2}$.

A selected MAE sample was placed on the horizontally oriented nonmagnetic platform. Magnetic field $B$ was applied to the sample by placing a NdFeB-type permanent magnet in size of $20 \times 20 \times 5 \mathrm{~mm}^{3}$ underneath the sample platform. The orientation of the field in the central region of the sample was perpendicular to the sample surface. The magnet was mounted on a vertical translation stage and the magnitude of the magnetic field in the sample region was varied by changing the distance between the magnet and the sample. Before the experiments, the setup was calibrated by measurement of the field strength at the sample position as a function of translation distance with a Hall sensor. The field was varied in the range from $B=5 \mathrm{mT}$ to $B=180 \mathrm{mT}$. During the embossing process, the sample was exposed to $B=180 \mathrm{mT}$. The stamp for embossing was fixed on another vertical translation stage and placed above the sample. Then, it was slowly moved towards the top surface of the sample and gently pressed into the sample. The translation was stopped about $15 \mu \mathrm{m}$ after the stamp had touched the surface. In the last part of the process, the stamp was carefully lifted back to its initial position. 
The observations revealed that only samples with $80 \mathrm{wt} \%$ of CIP could have retained the embossed pattern for several hours, while for samples with $70 \mathrm{wt} \%$ of CIP the pattern vanished in about $5 \mathrm{~min}$ after the embossing process, regardless of the applied magnetic field. Therefore, only samples with $80 \%$ of CIP were used for further experiments. These samples were named M1, M2 and M3 and had the following values of the shear storage modulus at $B=0: 7.1 \mathrm{kPa}(\mathrm{M} 1), 16.3 \mathrm{kPa}(\mathrm{M} 2)$ and $25.5 \mathrm{kPa}(\mathrm{M} 3)$, respectively.

Optical diffraction measurements were performed with a collimated beam from a He-Ne laser with the wavelength $\lambda=633 \mathrm{~nm}$ and output power of $5 \mathrm{~mW}$ (Uniphase, Manteca, CA, USA). The beam was linearly polarized in the direction parallel to the sample surface (TE polarization) and was impinging on the sample at the angle $\theta=67^{\circ}$ with respect to the surface normal. The transversal diameter of the beam on the surface was $1 \mathrm{~mm}$. The far-field diffraction pattern was projected onto the opaque screen placed around $30 \mathrm{~cm}$ behind the sample. It was imaged by the monochrome CCD camera (Blackfly BFLYU3-23S6M-C, FLIR, Wilsonville, OR, USA). The intensities $I_{0}, I_{-1}$ and $I_{+1}$ of the specular reflected beam (0th order diffraction peak) and the -1 st and the +1 st order diffraction peaks were analyzed. The intensities of higher-order peaks were negligible. The \pm 1 st order diffraction peaks were observed at the diffraction angles

$$
\gamma \approx \pm \frac{\lambda}{\Lambda \cos \theta}= \pm 3.1^{\circ}
$$

with respect to the direction of the specular reflection (see Figure 1). After background subtraction, the intensities of the three peaks were obtained by integration of the CCD signal within the three equal-sized areas corresponding to the area of the specular reflection. The diffraction efficiency was calculated as

$$
\eta_{m}=\frac{I_{m}}{I_{-1}+I_{0}+I_{+1}}, m=-1,+1 .
$$

By this definition, which is frequently used in experiments performed on scattering media, the absorption and scattering losses of the probe beam are disregarded. We observed that the value of $\eta_{-1}$ was sometimes somewhat larger than the value of $\eta_{+1}$. So, to enable more relevant comparison between different experiments, we usually consider average diffraction efficiency $\langle\eta\rangle=\left(\eta_{+1}+\eta_{-1}\right) / 2$.

Standard optical microscopy imaging (Nikon Optiphot2, Nikon, Tokyo, Japan) of the samples was performed with a long working distance objective with a $5 \times$ magnification. The magnet was kept under the sample all the time between the embossing and the imaging process. Optical profilometry measurements were performed with a 3D optical microscope (Bruker-ContourGT-K0, Billerica, MA, USA) using white-light interferometric objectives with $5 \times$ and $20 \times$ magnification. Cross-sectional profiles of the surface topography within the surface area of $400 \times 400 \mu \mathrm{m}^{2}$ were analyzed. The profilometer is based on scanning white-light interferometry, where the distance between the sample and the interferometric objective is automatically varied, while the corresponding micrographs showing the vertical displacement of interference fringes are recorded.

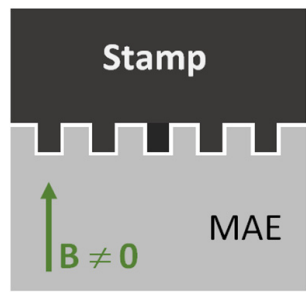

Write

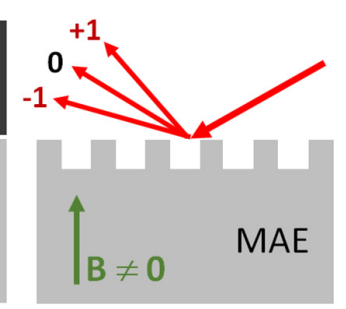

Read

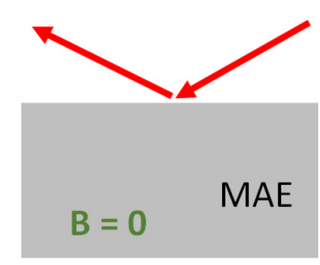

Erase

Figure 1. Schematic drawing of the writing (embossing), reading (optical diffraction) and erasure (transition to specular reflection) processes on the MAE surface. 
The basic idea for the performed investigations is schematically shown in Figure 1. The embossing process ("write" phase) takes place in the presence of a magnetic field and produces a surface micropattern of periodic channels that acts as an optical grating structure causing optical diffraction into the -1 st and the +1 st order diffraction peaks ("read" phase). This surface structure and the corresponding diffraction image persist so long as the magnetic field remains constant. When the magnetic field is removed, the diffraction vanishes and only specular reflection remains ("erase" phase).

\section{Results}

\subsection{Experimental Results}

An optical microscopy image of the sample surface after the embossing process is shown on the left side of Figure 2. A periodic pattern in the form of vertical stripes can be noticed. A smaller upper part of the image corresponds to the nonpatterned region. The right side of Figure 2. shows the optical diffraction setup. The laser spot on the sample surface is seen as a red ellipse at the bottom of the figure. A far-field diffraction pattern with three diffraction peaks is observed on the observation screen. The peaks are surrounded by a "halo" of diffuse scattering.
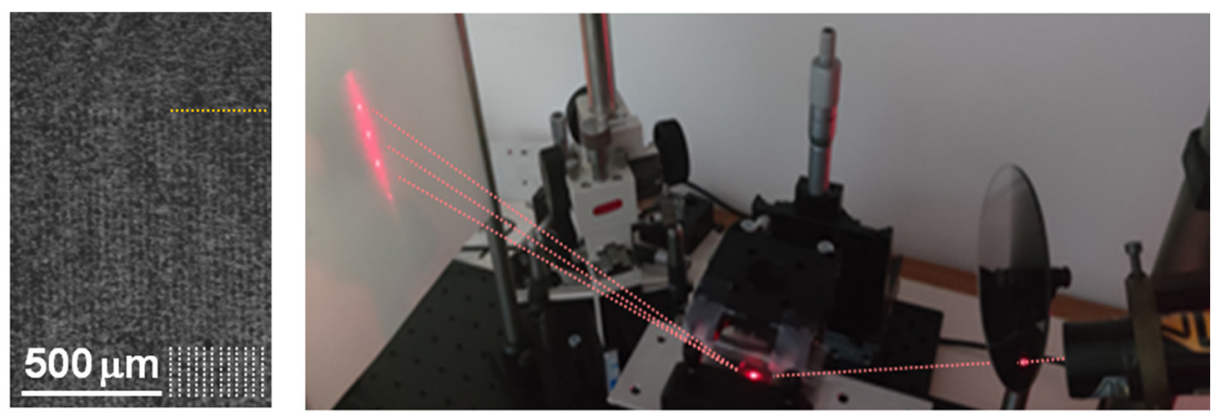

Figure 2. (left) Optical microscopy image of the micropatterned surface area. White vertical lines indicate grating lines. The border between the patterned and nonpatterned regions is indicated by a yellow horizontal line. (right) Photo of the experimental setup with a typical diffraction image projected onto a white screen. Red dotted lines indicate the paths of the incident and diffracted optical beams.

The cross-section of the diffraction pattern for sample M3 is shown in Figure 3a. To increase the visibility of the \pm 1 st order diffraction peaks, the intensity of the central (0th order) peak was attenuated for a factor of around 5 . The thick orange line corresponds to the diffraction pattern recorded a few minutes after the embossing and the thin black line to the diffraction pattern recorded four days after the embossing. Between the two measurements, the sample was constantly exposed to the magnetic field of $180 \mathrm{mT}$. The diffraction features remained practically the same, indicating that the embossed microstructure was stable over more than $90 \mathrm{~h}$. We also analyzed the decay of the diffraction efficiency after the magnet was quickly removed away from the sample. The result is shown in Figure $3 \mathrm{~b}$. The observed dependence was fitted to a two-exponential decay function. The obtained decay times are $\tau_{1}=0.68 \mathrm{~min}$ and $\tau_{2}=3.4 \mathrm{~min}$. 


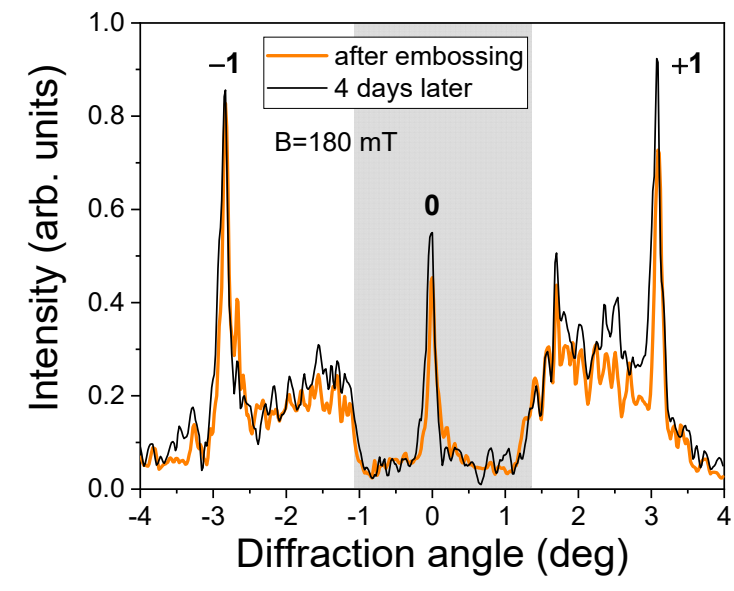

(a)

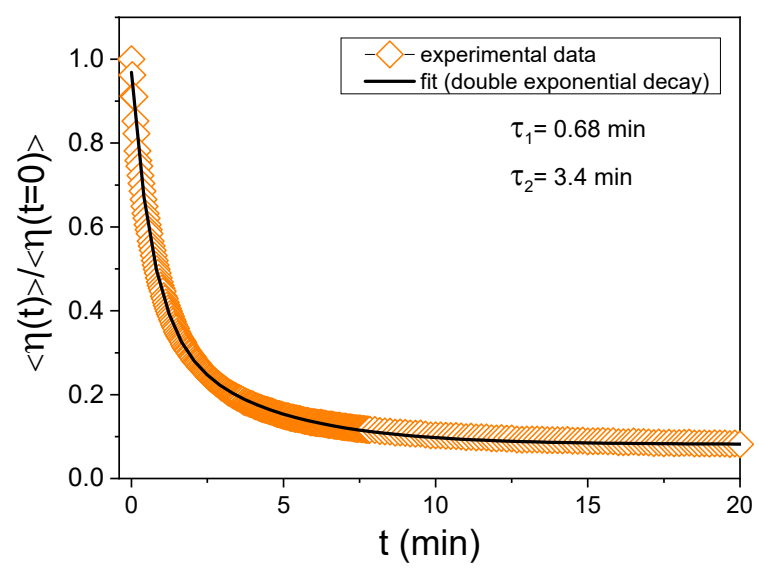

(b)

Figure 3. (a) Transversal profile of the far-field optical diffraction pattern recorded a few minutes (thick orange line) and four days (thin black line) after the embossing process in sample M3. To resolve more details, the optical signal in the central region (gray-shaded area) was attenuated for a factor of 5 with respect to other regions. The sample was constantly kept in the magnetic field $B=180 \mathrm{mT}$. (b) Time dependence of normalized diffraction efficiency $<\eta(t)>/<\eta(t=0)>$ after fast removal of the magnet. The solid line is a fit to a two-exponential decay function with decay times $\tau_{1}=0.68 \mathrm{~min}$ and $\tau_{2}=3.4 \mathrm{~min}$.

To examine the reconfigurability of the surface microstructure, we embossed and erased the pattern on the surface of the same sample several times. The orientation of the subsequent patterns was randomly varied. The observed optical diffraction patterns in two such experiments with sample M2 are shown in Figure 4a. The width of the diffraction peaks and the setting of diffuse scattering are slightly varied, while the ratio between the intensities of the -1 st and the 0th order peaks is practically the same. This result demonstrates good reproducibility of the embossed surface microstructure.

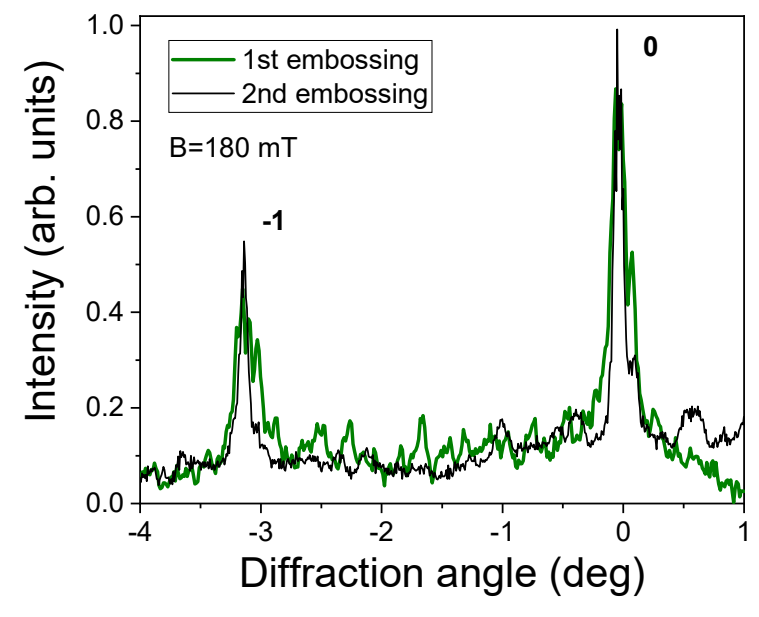

(a)

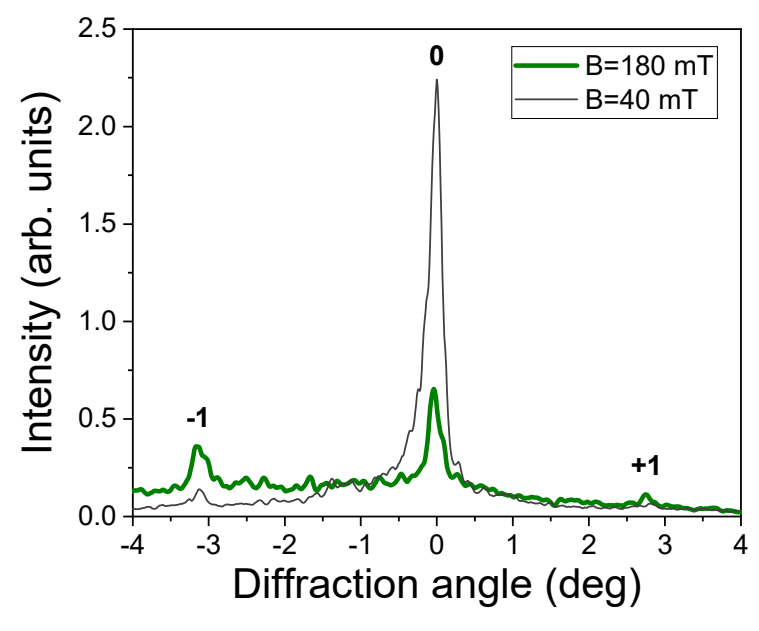

(b)

Figure 4. (a) Transversal profile of the far-field optical diffraction patterns observed as outcomes of two separate embossing processes in sample M2. Both diffraction measurements were performed at $B=180 \mathrm{~T}$. In between the two embossing episodes, the sample was removed from the magnetic field for several hours. (b) Far-field optical diffraction pattern observed at $B=180 \mathrm{mT}$ (thick green line) and at $B=40 \mathrm{mT}$ (thin black line). The value of $B$ was slowly reduced from $180 \mathrm{mT}$ to $40 \mathrm{mT}$ in steps of about $20 \mathrm{mT}$. 
In the next series of experiments, we investigated modifications of the diffraction efficiency during a step-by-step decrease of the magnetic field from $B=180 \mathrm{mT}$ to $B=0$. After every modification of the field, we let the structure stabilize for $10 \mathrm{~min}$ before the diffraction pattern was captured. Figure $4 \mathrm{~b}$ shows the diffraction patterns obtained at $B=180 \mathrm{mT}$ (green line) and $B=40 \mathrm{mT}$ (black line). The intensity of the 0th order peak increases with the decreasing field, while the intensities of the \pm 1 st order peaks decrease. Consequently, the diffraction efficiency decreases too. In addition to this, on these diffraction patterns also some asymmetry between the -1 st and the +1 st peak can be noticed.

Figure 5 a shows average diffraction efficiency $<\eta>$ as a function of magnetic field $B$ for sample M2. The value of $\langle\eta\rangle$ monotonically decreases with decreasing field and at $B=0$ practically vanishes. Figure $5 \mathrm{~b}$ gives a normalized diffraction efficiency $\left\langle\eta(B)>/\left\langle\eta\left(B_{\max }\right)>\right.\right.$ as a function of $B$ for all three investigated MAEs compositions. For the hardest sample (M3), a decrease of diffraction efficiency to $1 / 2$ of its initial value occurs at $B \sim 100 \mathrm{mT}$, while for the softest sample (M1), it occurs at $B \sim 60 \mathrm{mT}$. The medium-soft sample (M2) exhibits an intermediate behavior. This observation implies, as is expected, that the elasticity-driven erasure process is more efficient in hard than in soft samples. After the erasure process, if the magnetic field is increased again, the diffraction efficiency remains the same as at the end of the erasure process (these data are not shown).

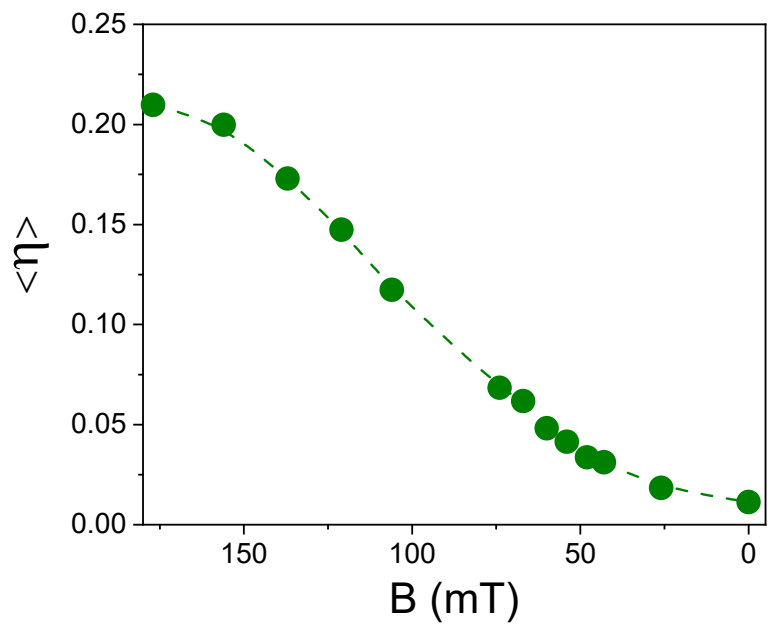

(a)

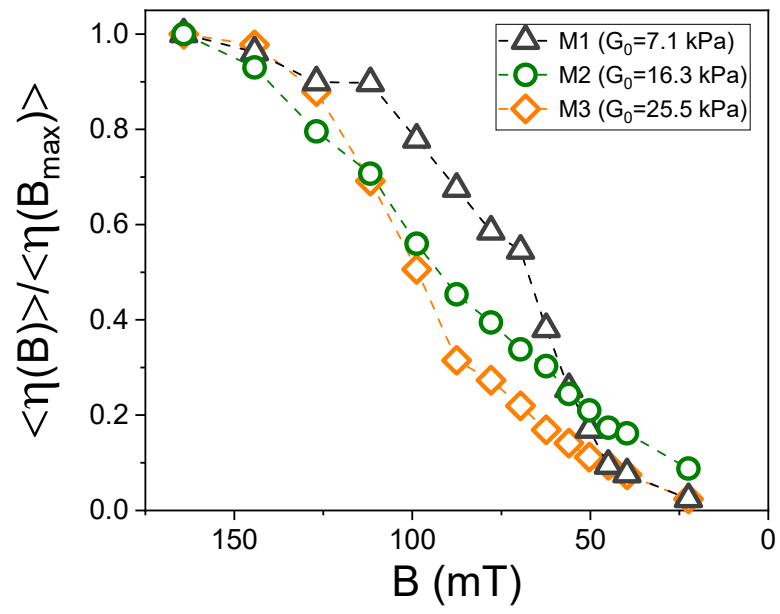

(b)

Figure 5. (a) Measured diffraction efficiency $<\eta>$ as a function of magnetic field $B$ for sample M2. (b) Normalized diffraction efficiency $<\eta(B)>/<\eta\left(B_{\max }\right)>$ as a function of magnetic field $B$ for three MAE samples with different matrix softness.

The last series of experiments considered surface analysis with 3D optical profilometry. Figure 6a. shows the surface topography of sample M1 imaged in the absence of a magnetic field. The surface is very flat, only some "craters" due to air bubbles can be noticed. Figure $6 \mathrm{~b}, \mathrm{c}$. show the surface of the same sample after the embossing process at two different magnifications. During the embossing and the subsequent imaging, the sample was constantly exposed to $B=180 \mathrm{mT}$. A periodic modulation of the surface topography is revealed. At the bottom of Figure $6 c$, a cross-sectional profile of surface features along the white horizontal line depicted in the topographical image is shown. The regions with a relatively flat surface landscape periodically exchange with the regions of very distorted surface structure. The distortions are in the vertical range of $\pm 10 \mu \mathrm{m}$. For comparison, Figure $6 \mathrm{~d}$ shows a topographical image and the cross-sectional profile of the stamp used for embossing. Periodic slabs with a height of $15 \mu \mathrm{m}$ can be clearly resolved. However, one can notice that there appear many "spikes" at the edge regions of the slabs. We assume that this is an artifact associated with the white-light interferometry that generally has difficulties resolving sharp edges. 


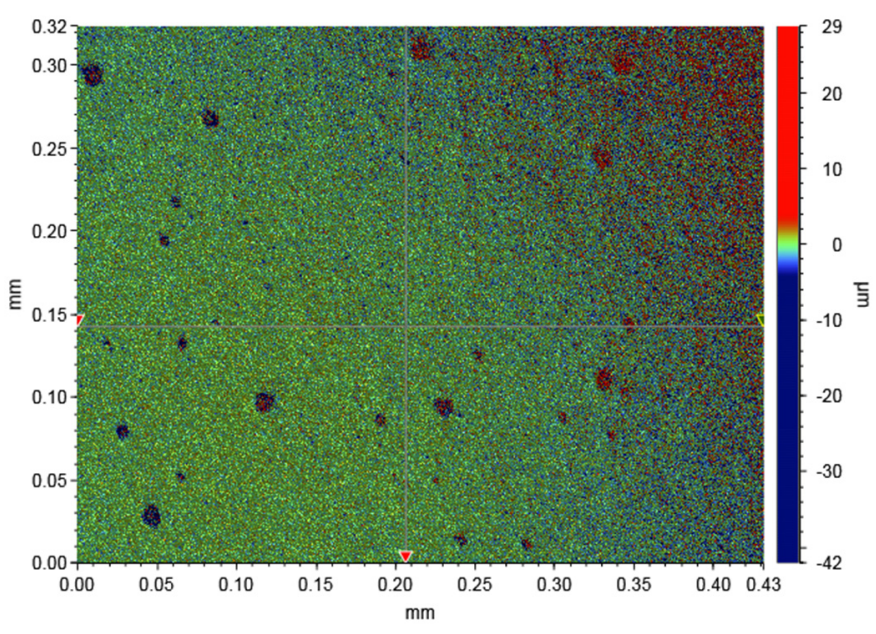

(a)
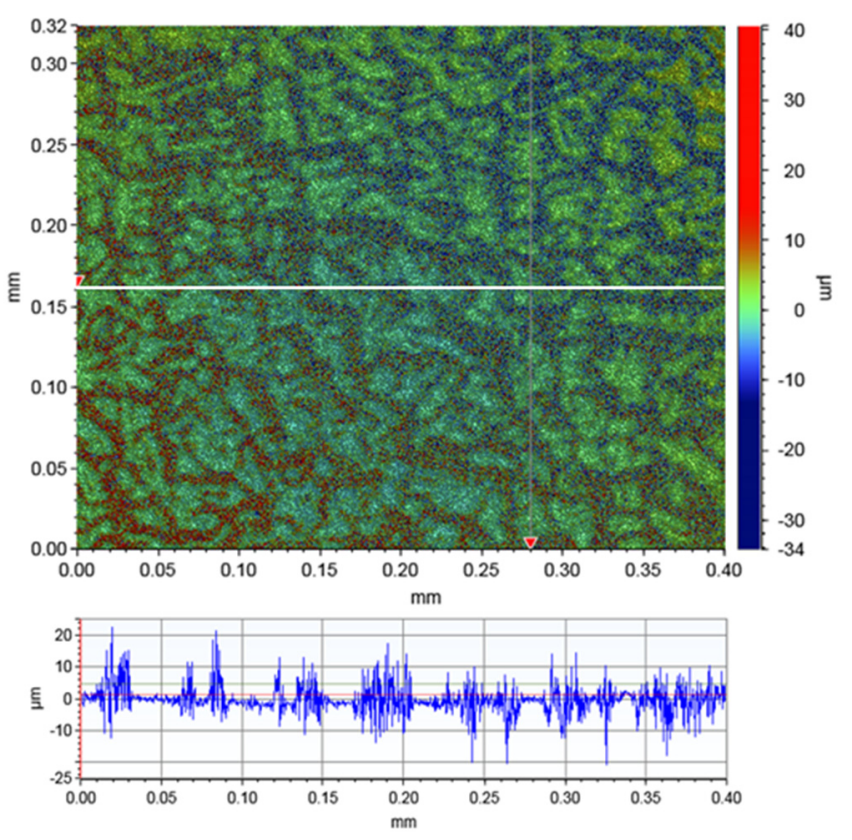

(c)

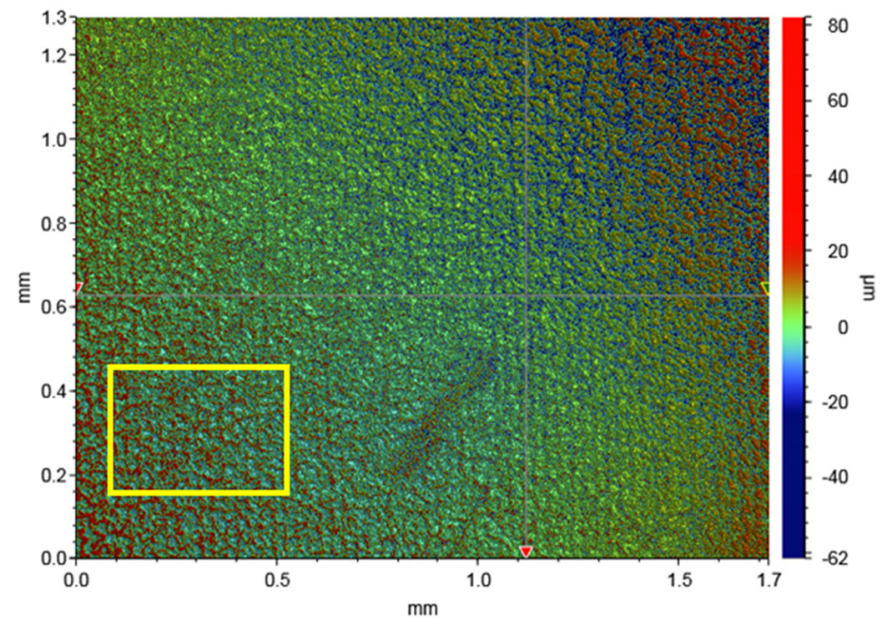

(b)
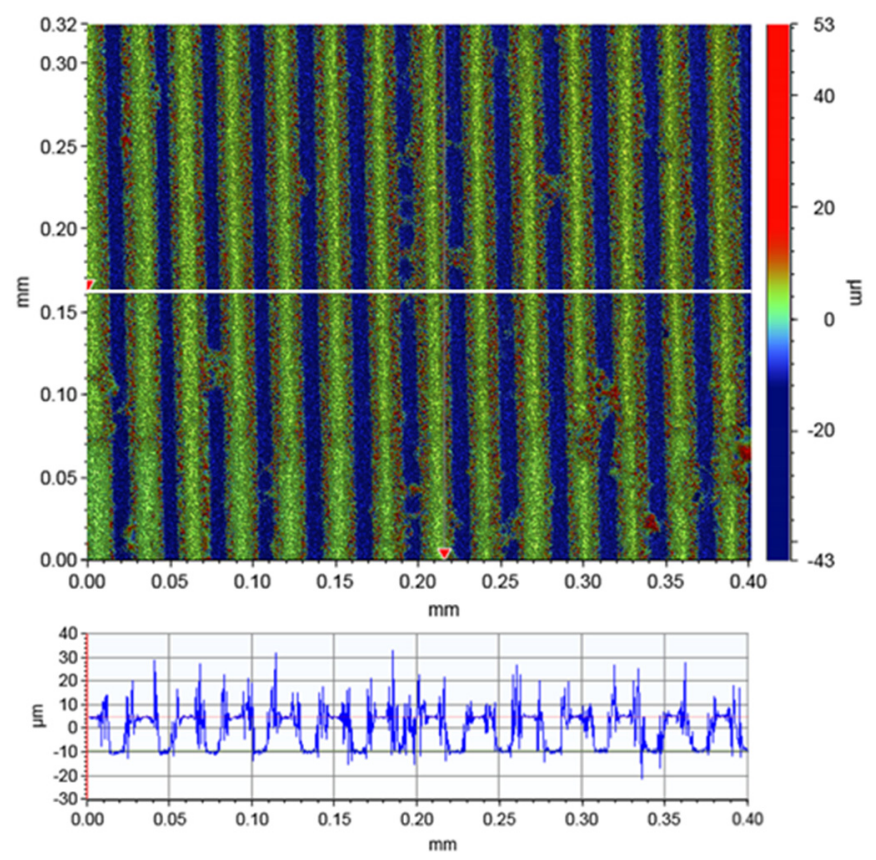

(d)

Figure 6. Optical profilometry images of (a) the surface of sample M1 before embossing in $B=0$ and (b,c) after embossing in $B=180 \mathrm{mT}$. (d) Optical profilometry image of the stamp used for embossing. Graphs at the bottom of (c,d) are cross-sections along the white lines depicted in topographical images. Yellow square in $(\mathbf{b})$ indicates the relative size of the surface areas shown in images $(\mathbf{a}, \mathbf{c}, \mathbf{d})$.

\subsection{Model of the Optical Diffraction Structure}

In accordance with the results of the 3D optical profilometry (Figure 6c), we propose to describe the imposed optical grating structure as a set of parallel stripes with two different amplitude reflectivity coefficients $\left(r_{1}\right.$ and $\left.r_{2}\right)$. The schematic drawing of the model is shown in Figure 7a. The stripes with larger roughness are supposed to have a smaller value of $r$, so we assume $r_{2}<r_{1}$. This is because larger roughness induces stronger spread optical reflection and consequently, less light is reflected in the direction of specular reflection, which effectively acts as a reduced reflectivity. 


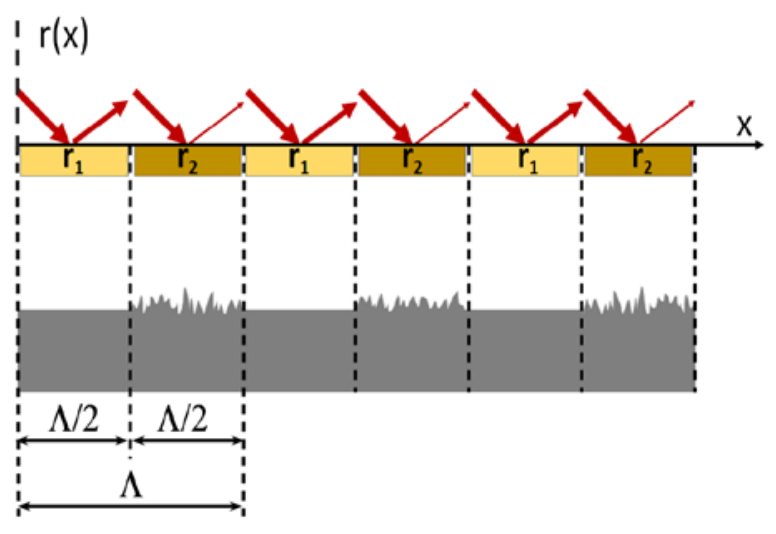

(a)

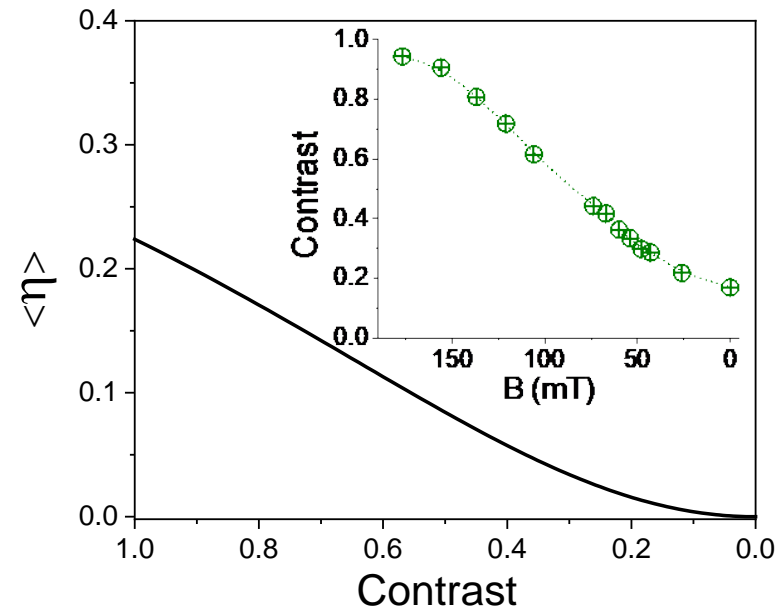

(b)

Figure 7. (a) Schematic drawing of the optical grating composed of parallel reflective stripes with amplitude reflectivity coefficients $r_{1}$ and $r_{2}<r_{1}$ (top drawing) and the corresponding MAE surface topography (bottom drawing). (b) Calculated dependence of diffraction efficiency $<\eta>$ of the \pm 1 st diffraction orders as a function of reflectivity contrast $c=\left(r_{1}-r_{2}\right) /\left(r_{1}+r_{2}\right)$. The inset gives the contrast of the grating lines for sample M2 as a function of magnetic field $B$ calculated on the basis of the experimental data shown in Figure 5a.

In the Fraunhofer diffraction theory, the diffraction pattern of such a structure is described as [51]:

$$
I(\gamma) \propto\left(\frac{\sin \left(\frac{N \omega \Lambda}{2}\right)}{\sin \left(\frac{\omega \Lambda}{2}\right)}\right)^{2}|f(\gamma)|^{2},
$$

where the first term is the structure factor in which $\omega=2 \pi \gamma \cos \theta / \lambda$ is the spatial frequency and $N$ is the number of illuminated grating periods, which in our setup was $\sim 85$ and $f(\gamma)$ is the form factor. The latter is calculated as

$$
f(\gamma)=\int_{0}^{\Lambda} a(x) e^{i \omega x} d x
$$

where $a(x)$ is the aperture function given as

$$
a(x)=\left\{\begin{array}{l}
r_{1} ; 0<x<\Lambda / 2 \\
r_{2} ; \Lambda / 2<x<\Lambda
\end{array} .\right.
$$

For such an aperture function, it follows:

$$
|f(\gamma)|^{2}=\left(\frac{\Lambda}{2}\right)^{2}\left(\frac{\sin \left(\frac{\omega \Lambda}{4}\right)}{\frac{\omega \Lambda}{4}}\right)^{2}\left[r_{1}^{2}+r_{2}^{2}+2 r_{1} r_{2} \cos \left(\frac{\omega \Lambda}{2}\right)\right] .
$$

The diffraction maxima appear when the denominator in the first term of the Equation (3) is zero, i.e., when the condition $\omega=m(2 \pi / \Lambda)$ is fulfilled, where $m=0, \pm 1, \pm 2, \ldots$ denotes the diffraction order. The corresponding values of $|f(\gamma)|^{2}$, which determine the intensities of the peaks, are:

$$
|f(\gamma)|_{m}^{2}=\left\{\begin{array}{c}
\left(\frac{\Lambda}{2}\left(r_{1}+r_{2}\right)\right)^{2} ; \mathrm{m}=0 \\
\left(\frac{\Lambda}{m \pi}\left(r_{1}-r_{2}\right)\right)^{2} ; \text { for odd } \mathrm{m} \\
0 \quad ; \text { for even } \mathrm{m}
\end{array} .\right.
$$


The diffraction efficiency as defined by Equation (2) is then given as

$$
\eta_{ \pm 1}=\frac{c^{2}}{2 c^{2}+(\pi / 2)^{2}}
$$

where $c$ is the reflectivity contrast defined as

$$
c=\frac{r_{1}-r_{2}}{r_{1}+r_{2}}
$$

In accordance with this model, it follows that $\eta_{-1}=\eta_{+1}$ and consequently $\langle\eta\rangle=\eta_{ \pm 1}$. The resulting dependence of $\langle\eta\rangle$ on $c$ as described by Equation (8) is shown as a solid line in Figure $7 \mathrm{~b}$. The value of $\langle\eta\rangle$ monotonically decreases with the decreasing contrast and becomes zero when $c=0$, which happens when surface roughness over the entire surface becomes homogeneous; therefore, the grating pattern vanishes.

Equation (8) can be inverted to calculate $c$ from the experimentally obtained values of $\langle\eta\rangle$. The resulting dependence of $c(B)$ for sample M2 is shown in the inset of Figure $7 \mathrm{~b}$. The contrast decreases with the decreasing value of $B$; however, it does not completely vanish at $B=0$. We observed that a complete erasure of the grating pattern spontaneously happens in about $24 \mathrm{~h}$ after the removal of the magnet from the sample. Alternatively, the erasure can be strongly stimulated by a repetitive approach and distancing of the magnet to/from the sample.

\section{Discussion}

Our results demonstrate that the shape memory effect of MAEs enables imprinting of temporary surface micropatterns, which exist as long as the magnetic field is present and are spontaneously erased after the field is removed. Subsequently, the field can be applied again and either the same or another pattern can be imprinted. The associated surface microstructure is hence reconfigurable. Our results also show that the readout of the imprinted micropattern can be conveniently realized by illuminating the surface with a coherent optical beam and recording its far-field optical diffraction image in the reflection grating configuration. This method enables analysis of the spatial as well as the temporal modifications of the surface pattern.

The observed optical diffraction phenomena and the obtained surface topography images reveal that the embossed surface pattern does not entirely resemble the shape of the stamp used for embossing. Instead of a structure of periodic surface channels, as depicted in Figure 1, we obtained a structure with periodically modulated surface roughness. This result is attributed to the fact that the thickness and the depth of the slabs in the stamp $(15 \mu \mathrm{m})$ are comparable to the size of the CIP microparticles (average diameter $4.5 \mu \mathrm{m}$ ) present in the material. Consequently, the spatial resolution of the embossing process is limited by the particle size. Better quality of the embossed replicas is, hence, to be expected if MAEs with smaller particles are used. Another effect that reduces the quality of the replicas is the adhesion of the stamp to MAE, which affects especially the detachment process of the stamp after the embossing. For softer MAE samples, we often noticed that small parts of the MAE material were torn away from the surface and remained trapped in the channels of the stamp. We found that this problem can be reduced by a slight tilting of the stamp during the embossing process. Further improvements are possible by surface coating of the stamp with a layer that decreases the adhesion.

The reported technical solutions for elastomeric reconfigurable optical diffraction gratings were primarily related to microfluidic applications [52-54]. They relied either on purely mechanical deformation of PDMS gratings $[55,56]$ for pressure and force measurements or on filling of a PDMS diffraction grating with a fluid sample for measuring the optical properties of fluid [52]. These technologies did not find wide application. Alternatively, combinations of liquid crystals with soft polymers have been employed to demonstrate light-responsive and electro-activated diffraction gratings [57]. In this work, we propose an 
alternative method for reconfiguring flexible diffraction gratings by the use of a magnetic field. Regardless of the above-mentioned imperfections observed in our experiments, the embossed grating structures induced optical diffraction with easily detectable and clearly resolvable diffraction maxima. This suggests that MAEs can be used for the fabrication of magnetically controllable diffractive optical elements (DOEs). Although DOEs are one of the most important and consequently also most researched units for manipulation of optical signals, reports on magnetically regulable DOEs are relatively rare. The majority of investigations involving soft materials are focused on phase modulation-type gratings whose refractive index is tuned with the magnetic field [58-61]. In contrast, magnetically regulable gratings reported in this work belong to the amplitude modulation-type gratings, which are more difficult to be realized. Reconfigurable ridge-type surface gratings can be fabricated also from conventional shape memory polymers, utilizing thermally regulable reversible shape changes [62-64]. These gratings can be erased by melting of crystalline regions within the sample. Their diffractive properties are similar to diffractive properties of our MAE gratings. However, most of the investigated gratings in conventional shape memory polymers have grating pitch in the range of the wavelengths of visible light, as the main focus of the associated research is tuning of the structural color $[65,66]$.

In addition to being regulable by magnetic fields, MAEs are also mechanically very deformable, so they offer an additional possibility to regulate diffractive optical properties with mechanical strain and vice-versa to regulate the strain via diffractive optical response. We hence believe that the investigated phenomena might have some interesting implications in soft matter photonics and other soft matter-based technologies. Furthermore, the embossing of a surface relief grating and a non-destructive observation of its dynamics in time-varying magnetic fields can be used as a novel experimental method for the investigation of the magnetic-field-induced plasticity on the micrometer scale.

\section{Conclusions}

It is shown that the field-induced shape memory effect in MAEs also works on the micrometer scale. However, it is not the relief which is transferred to the MAE surface, but the modulation of the surface roughness takes place. The surface appears to be smoothed out where the flat ridges of the stamp are pressed against the MAE surface. Nevertheless, the resulting effect can be utilized for realization of reconfigurable diffractive optical elements, because the reflection coefficients from smoothed and rough stripes differ. It is also observed that MAE samples with the higher content of iron filling performed better than the samples with the lower concentration of particles. The origin of this advantage of higher filled MAE sample is not completely clear yet, it may be related to the jamming phenomenon. Further research is required in order to optimize the composition of MAE samples with respect to the enhancement of the diffraction efficiency and to find effective procedures for fast erasure of embossed gratings.

Author Contributions: Conceptualization, I.D.-O. and M.S.; methodology, I.D.-O., M.K., I.A.B., L.C. and L.Č.; software, M.L., D.B., G.G. and L.C.; validation, M.L., D.B., G.G. and L.C.,; formal analysis, I.D.-O.; investigation, M.L., D.B. and G.G.; resources, M.K.; writing—original draft preparation, I.D.-O.; writing-review and editing, M.S., M.L. and I.D.-O.; visualization, I.D.-O.; supervision, I.D.-O., M.S. and M.K. All authors have read and agreed to the published version of the manuscript.

Funding: This research was funded by Slovenian Research Agency (ARRS) in the framework of the research programs P1-0192 and P2-0231, research project J1-3006 and bilateral research project BI-DE/20-21-016. The bilateral research project was co-funded by the German Academic Exchange Service (project no. 57513473). The work of G.G. and M.S. was partially funded by the Deutsche Forschungsgemeinschaft (DFG, German Research Foundation)_Project nos. 389008375, 437391117, 466920132. I.A.B. thanks The State Conference of Women and Equality Officers at Bavarian Universities (LaKoF Bayern) for a Ph.D. scholarship.

Institutional Review Board Statement: Not applicable.

Informed Consent Statement: Not applicable. 
Data Availability Statement: The data reported in this work are openly available in Mendeley at https:/ / doi.org/10.17632/6v4dx92bfm.1 (accessed on 15 December 2021). Additional data are available on request from the corresponding author.

Conflicts of Interest: The authors declare no conflict of interest.

\section{References}

1. Filipcsei, G.; Csetneki, I.; Szilagyi, A.; Zrinyi, M. Magnetic Field-Responsive Smart Polymer Composites. Adv. Polym. Sci. 2007, 206, 137-189. [CrossRef]

2. Abramchuk, S.; Kramarenko, E.; Stepanov, G.; Nikitin, L.V.; Filipcsei, G.; Khokhlov, A.R.; Zrínyi, M. Novel Highly Elastic Magnetic Materials for Dampers and Seals: Part I. Preparation and Characterization of the Elastic Materials. Polym. Adv. Technol. 2007, 18, 883-890. [CrossRef]

3. Samal, S.; Škodová, M.; Abate, L.; Blanco, I. Magneto-Rheological Elastomer Composites. A Review. Appl. Sci. 2020, 10, 4899. [CrossRef]

4. Kang, S.S.; Choi, K.; Nam, J.-D.; Choi, H.J. Magnetorheological Elastomers: Fabrication, Characteristics, and Applications Materials 2020, 13, 4597. [CrossRef]

5. Liu, T.; Xu, Y. Magnetorheological elastomers: Materials and applications. In Smart and Functional Soft Materials; Dong, X., Ed.; IntechOpen: London, UK, 2019; pp. 147-180. [CrossRef]

6. Bastola, A.K.; Paudel, M.; Li, L.; Li, W. Recent Progress of Magnetorheological Elastomers: A Review. Smart Mater. Struct. 2020, 29, 123002. [CrossRef]

7. Odenbach, S. (Ed.) Magnetic Hybrid-Materials: Multi-Scale Modelling, Synthesis, and Applications; De Gruyter: Berlin, Germany, 2021; ISBN 978-3-11-056963-6.

8. Díez, A.G.; Tubio, C.R.; Etxebarria, J.G.; Lanceros-Mendez, S. Magnetorheological Elastomer-Based Materials and Devices: State of the Art and Future Perspectives. Adv. Eng. Mater. 2021, 23, 2100240. [CrossRef]

9. Lee, S.; Yim, C.; Kim, W.; Jeon, S. Magnetorheological Elastomer Films with Tunable Wetting and Adhesion Properties. ACS Appl. Mater. Interfaces 2015, 7, 19853-19856. [CrossRef]

10. Sorokin, V.V.; Sokolov, B.O.; Stepanov, G.V.; Kramarenko, E.Y. Controllable Hydrophobicity of Magnetoactive Elastomer Coatings. J. Magn. Magn. Mater. 2018, 459, 268-271. [CrossRef]

11. Nanpo, J.; Kawai, M.; Mitsumata, T. Magnetic-Field Sensitivity for Magnetic Elastomers with Various Elasticities. Chem. Lett. 2016, 45, 785-786. [CrossRef]

12. Sorokin, V.V.; Belyaeva, I.A.; Shamonin, M.; Kramarenko, E.Y. Magnetorheological Response of Highly Filled Magnetoactive Elastomers from Perspective of Mechanical Energy Density: Fractal Aggregates above the Nanometer Scale? Phys. Rev. E 2017, 95, 062501. [CrossRef]

13. Belyaeva, I.A.; Kramarenko, E.Y.; Shamonin, M. Magnetodielectric Effect in Magnetoactive Elastomers: Transient Response and Hysteresis. Polymer 2017, 127, 119-128. [CrossRef]

14. Maman, M.; Ponsinet, V. Wettability of Magnetically Susceptible Surfaces. Langmuir 1999, 15, 259-265. [CrossRef]

15. Knieling, H.; Richter, R.; Rehberg, I.; Matthies, G.; Lange, A. Growth of Surface Undulations at the Rosensweig Instability. Phys. Rev. E 2008, 76, 066301. [CrossRef]

16. Psarra, E.; Bodelot, L.; Danas, K. Two-Field Surface Pattern Control via Marginally Stable Magnetorheological Elastomers. Soft Matter 2017, 13, 6576-6584. [CrossRef]

17. Cvek, M.; Mrlik, M.; Sevcik, J.; Sedlacik, M. Tailoring Performance, Damping, and Surface Properties of Magnetorheological Elastomers via Particle-Grafting Technology. Polymers 2018, 10, 1411. [CrossRef] [PubMed]

18. Sánchez, P.A.; Minina, E.S.; Kantorovich, S.S.; Kramarenko, E.Y. Surface Relief of Magnetoactive Elastomeric Films in a Homogeneous Magnetic Field: Molecular Dynamics Simulations. Soft Matter 2019, 15, 175-189. [CrossRef]

19. Li, R.; Li, X.; Chen, S.; Wang, X. The Effect of Magnetic Field on Surface Roughness of Magnetorheological Elastomers: A Theoretical Simulation. Proc. SPIE 2018, 10595, 105951J. [CrossRef]

20. Chen, S.; Dong, S.; Wang, X.; Li, W. Magneto-Induced Surface Morphologies in Magnetorheological Elastomer Films: An Analytical Study. Smart Mater. Struct. 2019, 28, 045016. [CrossRef]

21. Glavan, G.; Kettl, W.; Brunhuber, A.; Shamonin, M.; Drevenšek-Olenik, I. Effect of Material Composition on Tunable Surface Roughness of Magnetoactive Elastomers. Polymers 2019, 11, 594. [CrossRef] [PubMed]

22. Li, R.; Li, X.; Yang, P.; Liu, J.; Chen, S. The Field-Dependent Surface Roughness of Magnetorheological Elastomer: Numerical Simulation and Experimental Verification. Smart Mater. Struct. 2019, 28, 085018. [CrossRef]

23. Nadzharyan, T.A.; Stolbov, O.V.; Raikher, Y.L.; Kramarenko, E.Y. Field-Induced Surface Deformation of Magnetoactive Elastomers with Anisometric Fillers: A Single-Particle Model. Soft Matter 2019, 15, 9507-9519. [CrossRef] [PubMed]

24. Zhou, Y.; Huang, S.; Tian, X. Magnetoresponsive Surfaces for Manipulation of Nonmagnetic Liquids: Design and Applications. Adv. Funct. Mater. 2020, 30, 1906507. [CrossRef]

25. Glavan, G.; Salamon, P.; Belyaeva, I.A.; Shamonin, M.; Drevenšek-Olenik, I. Tunable Surface Roughness and Wettability of a Soft Magnetoactive Elastomer. J. Appl. Polym. Sci. 2018, 135, 46221. [CrossRef]

26. Yang, C.; Wu, L.; Li, G. Magnetically Responsive Superhydrophobic Surface: In Situ Reversible Switching of Water Droplet Wettability and Adhesion for Droplet Manipulation. ACS Appl. Mater. Interfaces 2018, 10, 20150-20158. [CrossRef] [PubMed] 
27. Watanabe, M.; Tanaka, Y.; Murakami, D.; Tanaka, M.; Kawai, M.; Mitsumata, T. Optimal Plasticizer Content for Magnetic Elastomers Used for Cell Culture Substrate. Chem. Lett. 2020, 49, 280-283. [CrossRef]

28. Chen, S.; Zhu, M.; Zhang, Y.; Dong, S.; Wang, X. Magnetic-Responsive Superhydrophobic Surface of Magnetorheological Elastomers Mimicking from Lotus Leaves to Rose Petals. Langmuir 2021, 37, 2312-2321. [CrossRef]

29. Krahn, J.; Bovero, E.; Menon, C. Magnetic Field Switchable Dry Adhesives. ACS Appl. Mater. Interfaces 2015, 7, $2214-2222$. [CrossRef]

30. Risan, J.; Croll, A.B.; Azarmi, F. Compliance Switching for Adhesion Control. J. Polym. Sci. Part B Polym. Phys. 2015, 53, 48-57. [CrossRef]

31. Testa, P.; Chappuis, B.; Kistler, S.; Style, R.W.; Heyderman, L.J.; Dufresne, E.R. Switchable Adhesion of Soft Composites Induced by a Magnetic Field. Soft Matter 2020, 16, 5806-5811. [CrossRef]

32. Li, R.; Wang, D.; Yang, P.; Tang, X.; Liu, J.; Li, X. Improved Magneto-Sensitive Adhesion Property of Magnetorheological Elastomers Modified Using Graphene Nanoplatelets. Ind. Eng. Chem. Res. 2020, 59, 9143-9151. [CrossRef]

33. Lee, D.-W.; Lee, K.; Lee, C.-H.; Kim, C.-H.; Cho, W.-O. A Study on the Tribological Characteristics of a Magneto-Rheological Elastomer. J. Tribol. 2012, 135, 014501. [CrossRef]

34. Lian, C.; Lee, K.-H.; Lee, C.-H. Friction and Wear Characteristics of Magneto-Rheological Elastomers Based on Silicone/Polyurethane Hybrid. J. Tribol. 2015, 137, 031607. [CrossRef]

35. Lian, C.; Lee, K.-H.; Lee, C.-H. Friction and Wear Characteristics of Magnetorheological Elastomer under Vibration Conditions. Tribol. Int. 2016, 98, 292-298. [CrossRef]

36. Lian, C.; Lee, K.-H.; Lee, C.-H. Effect of Temperature and Relative Humidity on Friction and Wear Properties of Silicone-Based Magnetorheological Elastomer. Tribol. Trans. 2018, 61, 238-246. [CrossRef]

37. Li, R.; Ren, D.; Wang, X.; Chen, X.; Chen, S.; Wu, X. Tunable Friction Performance of Magneto-Rheological Elastomer Induced by External Magnetic Field. J. Intell. Mater. Syst. Struct. 2018, 29, 160-170. [CrossRef]

38. Li, R.; Li, X.; Li, Y.; Yang, P.; Liu, J. Experimental and Numerical Study on Surface Roughness of Magnetorheological Elastomer for Controllable Friction. Friction 2020, 8, 917-929. [CrossRef]

39. Kravanja, G.; Belyaeva, I.; Hribar, L.; Drevensek-Olenik, I.; Jezeršek, M.; Shamonin, M. Tunable Drop Splashing on Magnetoactive Elastomers. Adv. Mater. Interfaces 2021, 8, 2100235. [CrossRef]

40. Nikitin, L.V.; Stepanov, G.V.; Mironova, L.S.; Gorbunov, A.I. Magnetodeformational Effect and Effect of Shape Memory in Magnetoelastics. J. Magn. Magn. Mater. 2004, 272-276, 2072-2073. [CrossRef]

41. Stepanov, G.V.; Abramchuk, S.S.; Grishin, D.A.; Nikitin, L.V.; Kramarenko, E.Y.; Khokhlov, A.R. Effect of a Homogeneous Magnetic Field on the Viscoelastic Behavior of Magnetic Elastomers. Polymer 2007, 2, 488-495. [CrossRef]

42. Stepanov, G.V.; Borin, D.Y.; Raikher, Y.L.; Melenev, P.V.; Perov, N.S. Motion of Ferroparticles inside the Polymeric Matrix in Magnetoactive Elastomers. J. Phys. Condens. Matter 2008, 20, 204121. [CrossRef]

43. Melenev, P.V.; Raikher, Y.L.; Rusakov, V.V.; Stepanov, G.V. Field-Induced Plasticity of Soft Magnetic Elastomers. J. Phys. Conf. Ser. 2009, 149, 012094. [CrossRef]

44. Melenev, P.; Raikher, Y.; Stepanov, G.; Rusakov, V.; Polygalova, L. Modeling of the Field-Induced Plasticity of Soft Magnetic Elastomers. J. Intell. Mater. Syst. Struct. 2011, 22, 531-538. [CrossRef]

45. Stepanov, G.; Kramarenko, E.; Semerenko, D. Magnetodeformational Effect of the Magnetoactive Elastomer and Its Possible Applications. J. Phys. Conf. Ser. 2013, 412, 2031. [CrossRef]

46. Stolbov, O.; Raikher, Y. Mesostructural Origin of the Field-Induced Pseudo-Plasticity Effect in a Soft Magnetic Elastomer. IOP Conf. Ser. Mater. Sci. Eng. 2019, 581, 012003. [CrossRef]

47. Shamonin, M.; Kramarenko, E.Y. Chapter 7-Highly Responsive Magnetoactive Elastomers. In Novel Magnetic Nanostructures; Elsevier: Amsterdam, The Netherlands, 2018. [CrossRef]

48. Testa, P.; Style, R.W.; Cui, J.; Donnelly, C.; Borisova, E.; Derlet, P.M.; Dufresne, E.R.; Heyderman, L.J. Magnetically Addressable Shape-Memory and Stiffening in a Composite Elastomer. Adv. Mater. 2019, 31, e1900561. [CrossRef] [PubMed]

49. Prem, N.; Sindersberger, D.; Striegl, B.; Böhm, V.; Monkman, G.J. Shape Memory Effects Using Magnetoactive Boron-Organo-Silicon Oxide Polymers. Macromol. Chem. Phys. 2020, 221, 2000149. [CrossRef]

50. Saveliev, D.V.; Belyaeva, I.A.; Chashin, D.V.; Fetisov, L.Y.; Romeis, D.; Kettl, W.; Kramarenko, E.Y.; Saphiannikova, M.; Stepanov, G.V.; Shamonin, M. Giant Extensional Strain of Magnetoactive Elastomeric Cylinders in Uniform Magnetic Fields. Materials 2020, 13, 3297. [CrossRef]

51. Fowles, G.R. Introduction to Modern Optics; Holt, Rinehart and Winston: New York, NY, USA, 1968.

52. Schueller, O.J.A.; Duffy, D.C.; Rogers, J.A.; Brittain, S.T.; Whitesides, G.M. Reconfigurable Diffraction Gratings Based on Elastomeric Microfluidic Devices. Sens. Actuators A Phys. 1999, 78, 149-159. [CrossRef]

53. Hosokawa, K.; Hanada, K.; Maeda, R. A Polydimethylsiloxane (PDMS) Deformable Diffraction Grating for Monitoring of Local Pressure in Microfluidic Devices. J. Micromech. Microeng. 2001, 12, 1. [CrossRef]

54. Yu, J.Q.; Yang, Y.; Liu, A.Q.; Chin, L.K.; Zhang, X.M. Microfluidic Droplet Grating for Reconfigurable Optical Diffraction. Opt. Lett. 2010, 35, 1890-1892. [CrossRef]

55. Grzybowski, B.A.; Qin, D.; Whitesides, G.M. Beam Redirection and Frequency Filtering with Transparent Elastomeric Diffractive Elements. Appl. Opt. 1999, 38, 2997-3002. [CrossRef] [PubMed] 
56. Grzybowski, B.; Qin, D.; Haag, R.; Whitesides, G.M. Elastomeric Optical Elements with Deformable Surface Topographies: Applications to Force Measurements, Tunable Light Transmission and Light Focusing. Sens. Actuators A Phys. 2000, 86, 81-85. [CrossRef]

57. Guglielmelli, A.; Nemati, S.; Vasdekis, A.E.; Sio, L.D. Stimuli Responsive Diffraction Gratings in Soft-Composite Materials. J. Phys. D Appl. Phys. 2018, 52, 053001. [CrossRef]

58. Chen, C.-Y.; Tsai, T.-R.; Pan, C.-L.; Pan, R.-P. Room Temperature Terahertz Phase Shifter Based on Magnetically Controlled Birefringence in Liquid Crystals. Appl. Phys. Lett. 2003, 83, 4497-4499. [CrossRef]

59. Chen, C.-Y.; Hsieh, C.-F.; Lin, Y.-F.; Pan, R.-P.; Pan, C.-L. Magnetically Tunable Room-Temperature $2 \pi$ Liquid Crystal Terahertz Phase Shifter. Opt. Express 2004, 12, 2625-2630. [CrossRef] [PubMed]

60. Zhang, L.; Fan, Y.-X.; Liu, H.; Han, X.; Lu, W.-Q.; Tao, Z.-Y. A Magnetically Tunable Non-Bragg Defect Mode in a Corrugated Waveguide Filled with Liquid Crystals. Phys. Lett. A 2018, 382, 1000-1005. [CrossRef]

61. Bošnjaković, D.; Sebastián, N.; Drevenšek-Olenik, I. Magnetically Tunable Liquid Crystal-Based Optical Diffraction Gratings. Polymers 2020, 12, 2355. [CrossRef] [PubMed]

62. Tippets, C.A.; Li, Q.; Fu, Y.; Donev, E.U.; Zhou, J.; Turner, S.A.; Jackson, A.-M.S.; Sheares Ashby, V.; Sheiko, S.S.; Lopez, R. Dynamic Optical Gratings Accessed by Reversible Shape Memory. ACS Appl. Mater. Interfaces 2015, 7, 14288-14293. [CrossRef]

63. Li, Z.; Sun, R.; Rahman, M.A.; Feng, J.; Olah, A.; Baer, E. Scaling Effect on the Optical Properties of Patterned Nano-Layered Shape Memory Films. Polymer 2019, 167, 182-192. [CrossRef]

64. Zhang, F.; Xia, Y.; Liu, Y.; Leng, J. Nano/Microstructures of Shape Memory Polymers: From Materials to Applications. Nanoscale Horiz. 2020, 5, 1155-1173. [CrossRef]

65. Schauer, S.; Baumberg, J.J.; Hölscher, H.; Smoukov, S.K. Tuning of Structural Colors Like a Chameleon Enabled by Shape-Memory Polymers. Macromol. Rapid Commun. 2018, 39, 1800518. [CrossRef] [PubMed]

66. Wang, Y.; Zhai, Y.; Villada, A.; David, S.N.; Yin, X.; Xiao, J. Programmable Localized Wrinkling of Thin Films on Shape Memory Polymers with Application in Nonuniform Optical Gratings. App. Phys. Lett. 2018, 112, 251603. [CrossRef] 